2019-07-08

Proof-of-concept modular robot platform for cauliflower harvesting

Klein, FB

http://hdl.handle.net/10026.1/14874

10.3920/978-90-8686-888-9_97

Precision agriculture '19

Wageningen Academic Publishers

All content in PEARL is protected by copyright law. Author manuscripts are made available in accordance with publisher policies. Please cite only the published version using the details provided on the item record or document. In the absence of an open licence (e.g. Creative Commons), permissions for further reuse of content should be sought from the publisher or author. 


\title{
Proof-of-concept Modular Robot Platform for Cauliflower Harvesting
}

\author{
F. B. Klein ${ }^{1}$, A. Wilmot ${ }^{1}$, V. F. de Tejada ${ }^{1}$, B.L. Rodriguez ${ }^{1}$, I. Requena ${ }^{3}$, S. Busch ${ }^{1}$, A. \\ Rondepierre $^{1}$, T. Auzeeri ${ }^{1}$, T. Sauerwald ${ }^{3}$, W.F.P. Andrews ${ }^{1}$, H. Rihan ${ }^{2}$, M.P. Fuller ${ }^{2}$ \\ and M. F. Stoelen ${ }^{1,3}$ \\ ${ }^{1}$ School of Computing Electronics and Mathematics, University of Plymouth, UK \\ ${ }^{2}$ School of Biological and Marine Sciences, University of Plymouth, PL4 8AA, UK \\ ${ }^{3}$ Fieldwork Robotics Ltd., PL4 8AA, UK \\ frederico.klein@plymouth.ac.uk
}

\begin{abstract}
This paper presents a proof-of-concept platform for demonstrating robotic harvesting of summer-varieties of cauliflower, and early tests performed under laboratory conditions. The platform is designed to be modular and has two dexterous robotic arms with variablestiffness technology. The bi-manual configuration enables the separation of grasping and cutting behaviours into separate robot manipulators. By exploiting the passive compliance of the variable-stiffness arms, the system can operate with both grasping and cutting tool close to the ground. Multiple 3D vision cameras are used to track the cauliflowers in real-time, and to attempt to assess the maturity. Early experiments with the platform in the laboratory highlight the potential and challenges of the platform.
\end{abstract}

Keywords: robotics, selective harvesting, variable-stiffness actuators, cauliflowers.

\section{Introduction}

The cost of rural workforce labour drives automation. A broad range of harvesting robot designs have been investigated over the last 30 years to enhance selective picking operations in agriculture. To name a few recent examples, in the EU-funded CROPS (Clever Robots for Crops) project, an autonomous robot platform for harvesting sweetpeppers in greenhouses was developed. The design concept implied a custom-design 9 degree-of-freedom (DOF) manipulator, 2 types of end-effectors, several RGB and depth cameras and a lighting rig. The overall performance of the robot system on simplified experiments was evaluated under typical crop environment conditions (Bac et al. 2017). A proof-of-concept robot system aimed at picking apples in modern orchards was investigated by Davidson et al. (2016). The robotic design comprised a custom 6 DOF manipulator mounted on a travelling vehicle and a tendon-driven end-effector. A global vision system consisting of an RGB camera and a depth camera was used for detection and 3D location of the fruit. Laboratory testing was conducted, and the performance evaluation was discussed. Despite the wide range of robotic designs explored, versatile robotic solutions and extensive field experiments along with performance evaluations are required to make harvesting robots commercially available in agricultural environments. Most harvesting operations for Brassica crops are still performed with manual labour, which drives up the costs for the farmer. Large combined harvesters for broccoli and cabbage are available, but the current technology has several drawbacks. First, it will harvest all crops in one part of the field at any time, mature or not, leading to extensive waste. No selective harvesters are currently commercially available. For broccoli, there is ongoing research on 3D vision (Kusumam et al., 2017) and harvesting (Wageningen, 2018), and some industrial testing is underway (KMS projects, 2018). Cauliflower are more challenging due to the extensive foliage, and the difficulty in reaching the curd. The 
potential economic benefit to the producer is a considerable reduction in costs compared to manual labour, less hygiene issues and potentially less waste.

In this paper, a proof-of-concept platform is presented that, when complete, will aim to reproduce all the steps in cauliflower harvesting, namely identifying maturity, grasping the curd, cutting it from its stem and placing on to a moving platform for packaging.
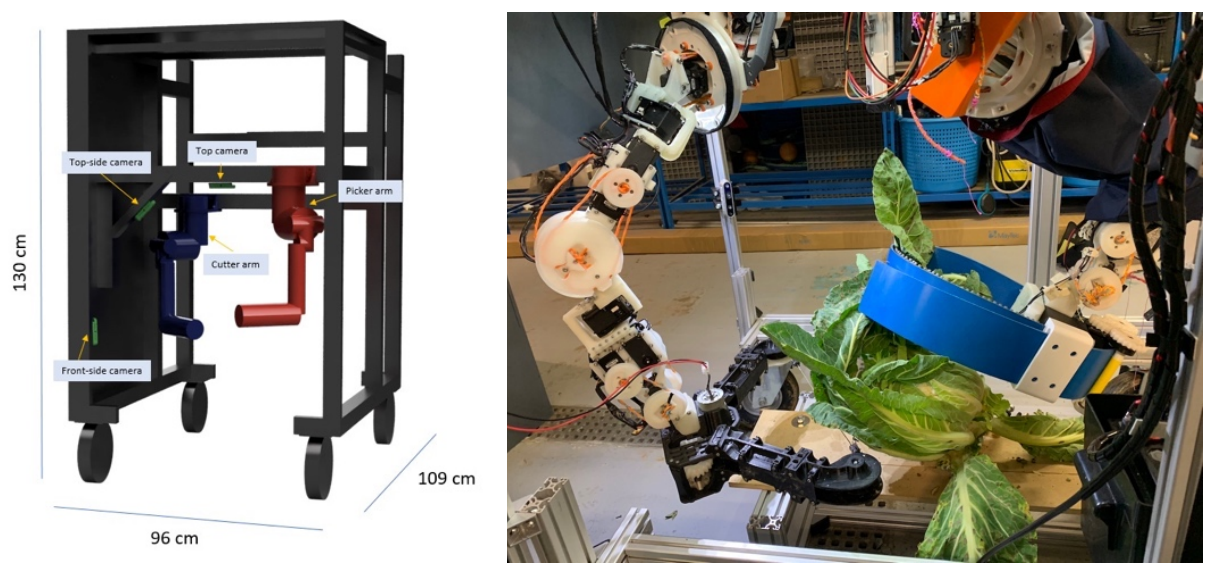

Figure 1: Left: Schematic render of the robotic cauliflower harvester color-coded for clarity. Red robotic arm is the cutting arm. Blue is picker arm. Green are the three D400 Intel Realsense cameras. Right: Image of cutting and gripping arm during lab testing.

\section{Materials and methods}

\section{Mobile test platform}

The robotic platform is shown in Figure 1. It consists of an inverted $U$ extruded aluminium frame with caster-wheels, fitted with two dexterous variable-stiffness robotic arms, and sensors. The arms are heavily modified versions of the open-source GummiArm (Stoelen et al., 2016), see Figure 2. Each arm is made up of three major sections, the shoulder, the upper arm and the lower arm, where an end-effector for a specialised task can be attached. The movement of the different sections of the arm is realised by employing an agonist-antagonist tendon pulley system. That is, by emulating the pairs of opposing muscles found around joints in the human body. This enables control of movement and stiffness (through co-contraction of opposing actuators). As earlier versions of the GummiArm were used for demonstrations or selective harvesting of delicate and light fruits, the payload needed to be increased for selective harvesting of cauliflowers. This was done by implementing a bi-directional set-up of the pulley system for the most demanding joints in all three sections. End-effectors and the major sections can be connected effortlessly, due to a dovetail connection system, which makes it easy to remove them. One of its robotic arms is fitted with a cutting end-effector and another fitted with a picking end-effector. Additionally, it has 3 RGB-D cameras to perform the visual tasks necessary to control the state machine, a top mounted white LED array and a dual power system that can run on either batteries or mains. 

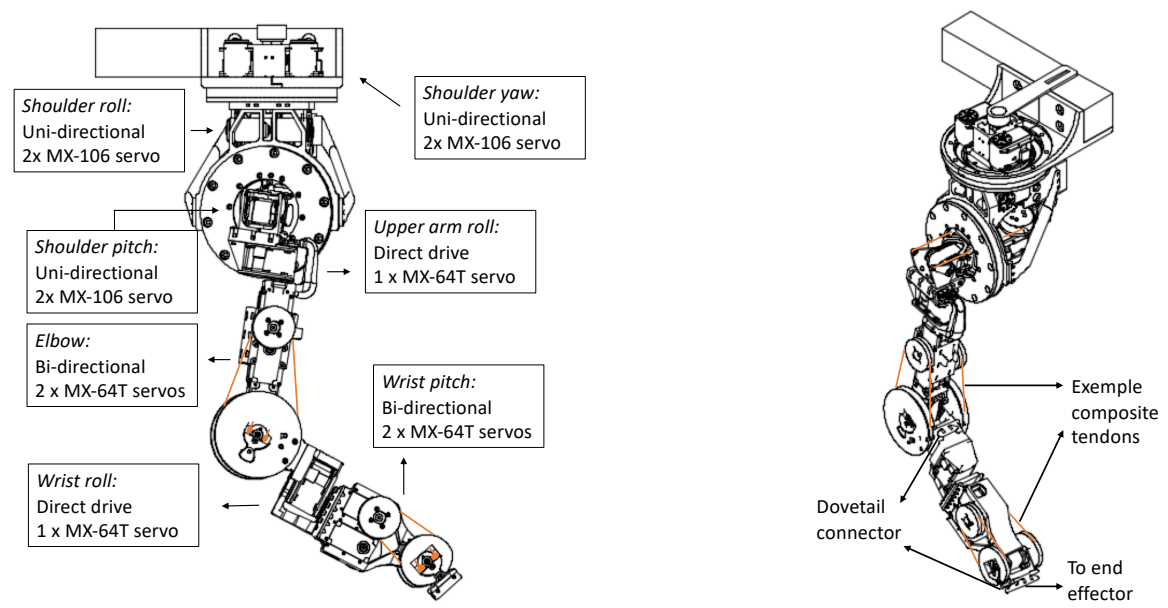

Figure 2: Two views of the modified version of the 7 jointed variable-stiffness GummiArm (Stoelen et al., 2016) in development for cutting and picking cauliflowers.

\section{Cutting end-effector}

For the cutting mechanism a reciprocating motion of the abrasive wire at the cutting interface is achieved by a single DC motor that drives a compound spur-gear-train with two cable-coupled output linkages, producing four-times the torque of the motor pinion. Separate $3 \mathrm{~mm}$ diameter flexible steel cables are fastened to the output linkages, fed through the hollow-space in the nearest limb, curved along the pulleys in the distal portion of the limbs, and ultimately connected to either end of the $2 \mathrm{~mm}$ diameter stainless-steel braided abrasion wire at the cutting interface: this couples the motion of the output linkages. The abrasive wire bifurcates the target by grinding through plant-matter, as opposed to slicing it; this makes the system inherently safer for use around human operators and livestock. The cutting end-effector weighs roughly $1.5 \mathrm{~kg}$, and is wide enough to cut cauliflowers with all leaves on. For emulating and quantifying the operation of the cutting end-effector, the cutting arm was controlled via teleoperation. The teleoperation was done with a SpaceNavigator (3DConnexion, US) joystick.

Gripping end-effectors

For the task of gripping the cauliflower, there are two major gripper designs: the belt gripper and the three fingered gripper. The belt gripper consists of a bendy, but stable 3ply PVC belt and its driving mechanism. The gripper will approach the cauliflower from above, push down any distant foliage, and enclose the cauliflower including its leaves. Gripping the leaves by tightening the belt provides an even pressure on the crop, reducing the risk of damage. The soft-fingered gripper consists of a modular base shape and three ends, driving the fingers with a rack and rail system. This makes it possible to independently adjust the torque needed or the spread needed for the crop to harvest. The gripper can approach the curd from any angle and will also grip a crop through foliage.

The arms are controlled using a software framework based on ROS MoveIt! (Sucan, 2018) also used for other applications such as tomato and raspberry picking, previously and currently being explored by the authors' group (Stoelen et al., 2018) (University of Plymouth, 2018). The soft end-effectors in an open position would be moved to the best estimate or the curd's centre in open loop, and by having it grasp around anything inside its grasping volume. Here a useful design feature of the passively compliant arm is exploited by setting the arm to be fully compliant/soft in every joint. This means it will 
no longer try to oppose any passive deflection of the end-effector caused by the beltgripper tightening its grip around the rooted cauliflower head.

\section{Computer Vision}

In the approach presented, the vision subsystem oversees two different tasks, namely i) detecting where a cauliflower is present in the 3D space, and ii) whether it is a cauliflower that should be picked or not. The cameras chosen were the Intel Realsense D400 series (Intel, 2018) due to the its low cost, high resolution and speed, allowing for a standard cross platform, cross application solution. To control lighting a set of ten $0.9 \mathrm{~m}$ long white LED strips, was evenly placed underneath the top of a fully covered platform.

A critical challenge in detecting cauliflowers is the partial or total occlusion of the curd by its leaves. The leaf growth pattern in Brassica's were exploited, namely that its leaves grow from the stem in a radial fashion and that the curd is on the top of this stem. To detect maturity curd flower separation was employed. A developing cauliflower has a very tight curd with no gaps and holes, and an overmatured one presents significant gaps in the curd. As such, the identification and measurement of the dark section in the curd of the vegetable can indicate ripeness, and the harvesting can be done. A diagram of curd and maturity detection can be seen in Figure 3.

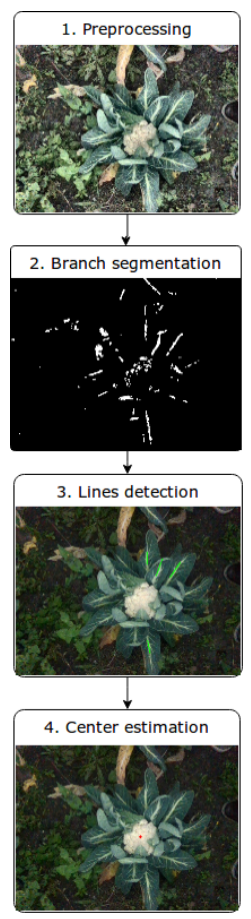

a)

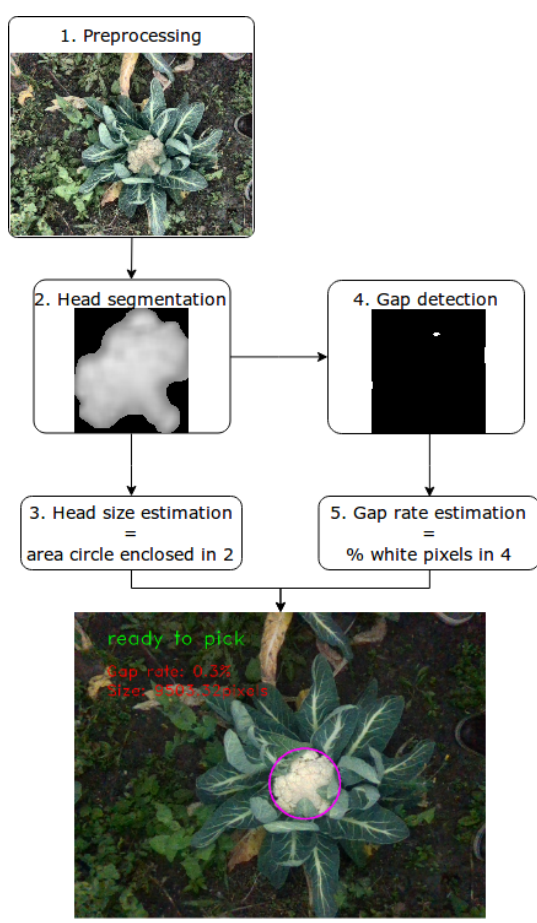

b)

Figure 3: Overview of vision algorithms for detection of a) curd, and b) maturity.

\section{Results}

In order to test the vision algorithms as an independent part of the whole system, a small dataset of cauliflowers of different varieties was manually collected. This was done using both the D415 and the D435 cameras and the images were collected with colour, infrared and depth channels. Each plant was imaged multiple times from different angles, at different times of day under different weather conditions, between March and May of 2018 on various occasions, mainly on a small university garden in the University of 
Plymouth campus and once in a commercial farm in Cornwall, UK and consisted mostly of around 5-10 plants of 3-4 different varieties, each plant being imaged from 6 to 50 times (median $=20$, average $=24.16, \sigma=13.54)$, resulting in a total of 1015 RGB images, most of which presented an exposed curd when mature. The results were qualitative, considering the great variability of the photos taken (different varieties, inconsistent lighting conditions, different cameras). The imaging was very different from the imaging obtained from the rig cameras. See Figure 4 and 5 for example results on localisation of centre and basic maturity estimation.

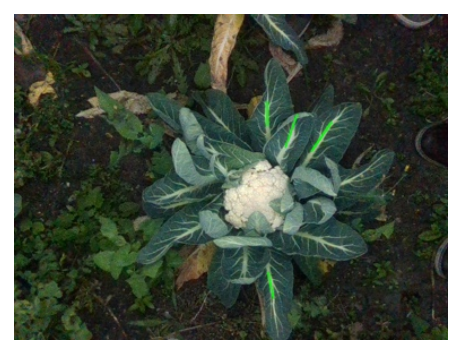

a)

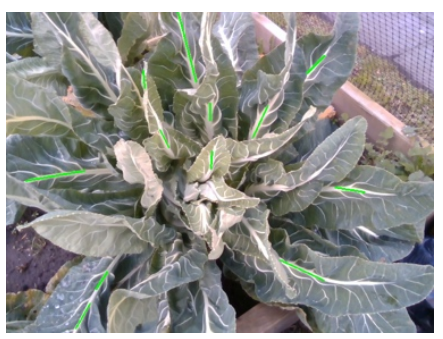

c)

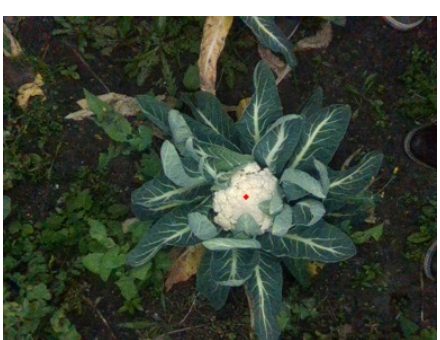

b)

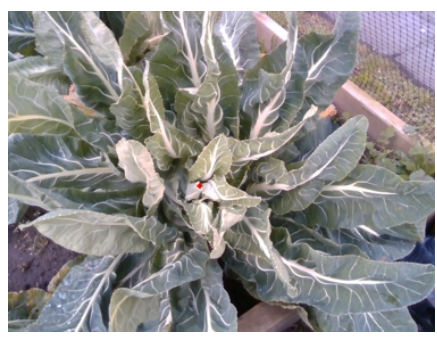

d)

Figure 4. Example results from localisation of the centre of the cauliflower curd, a) leaf vein detection (light green lines) for an open curd cauliflower, b) centre (red dot) from extrapolated intersection of those lines, c) a completely occluded curd with leaf veins detected, and d) centre extrapolated from vein lines for occluded curd (in red).

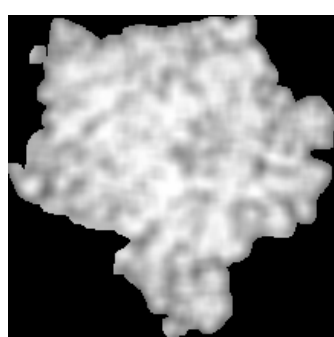

a)

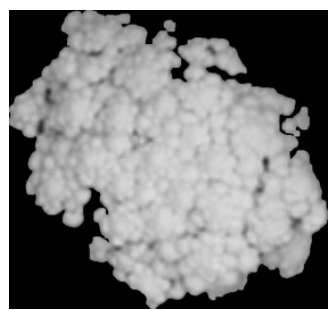

d)

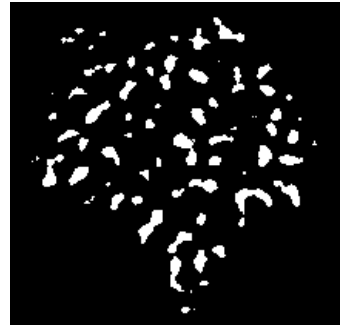

b)

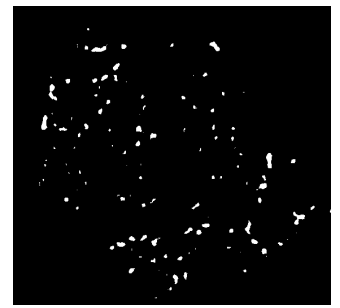

e)

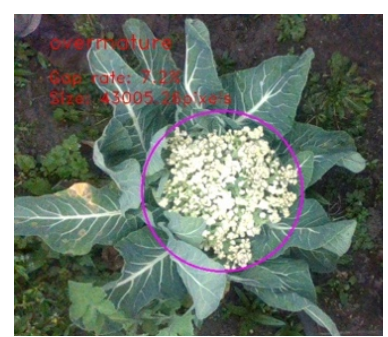

c)

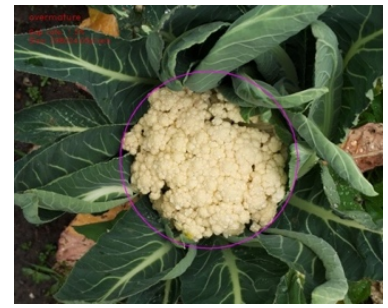

f) 
Figure 5. Example results from basic maturity estimation based on gap detection, a) mask focused on the exposed curd, b) gap detection, c) visible gaps in overly matured curd, d) masking on the exposed curd of younger cauliflower, e) less prominence of white spots in this example, and f) less visible gaps on this less overmatured curd.

For the cutting tests it was important that the robotic arm could perform the whole movement, approximately $0.3 \mathrm{~m}$ at the base of the cauliflower. The arm also needed to produce sufficient correction forces to maintain the direction of the cut. An image of the cutting setup can be seen in Figure 6. A force-sensitive surface was used, with a oneaxis load-cell. The resulting cut cauliflower curd can be seen in Figure 6c.

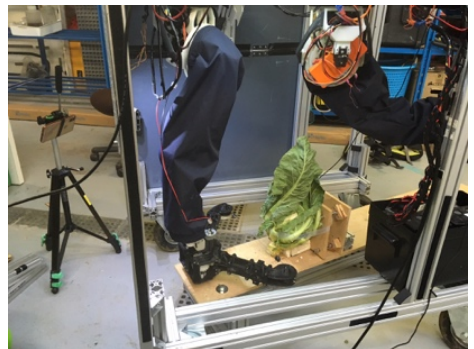

a)

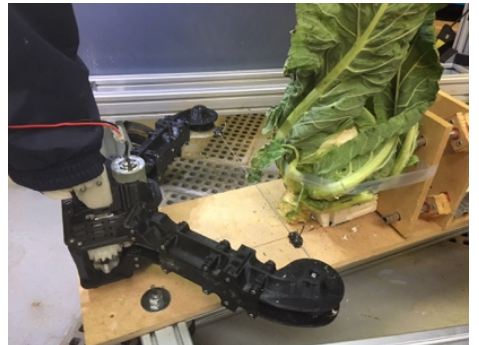

b)

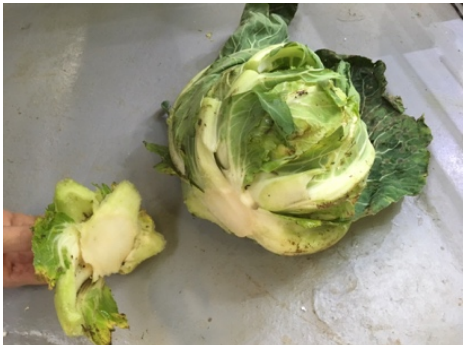

c)

Figure 6. Experiment setup for teleoperated cutting tests with force-sensitive surface, a) platform with stabilized cauliflower, b) wire cutter and force-sensitive surface, and c) the resulting cut in the cauliflower.

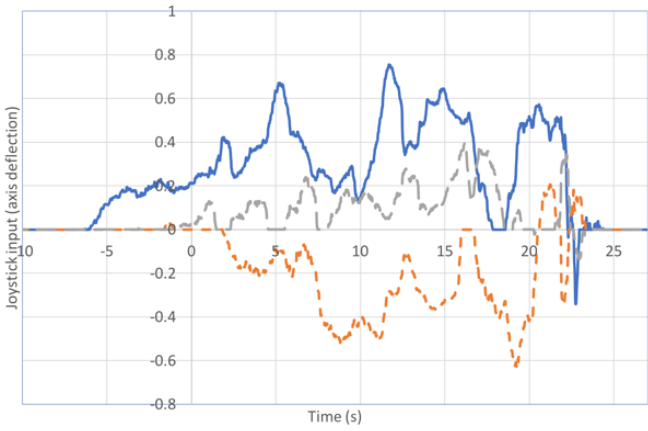

a)

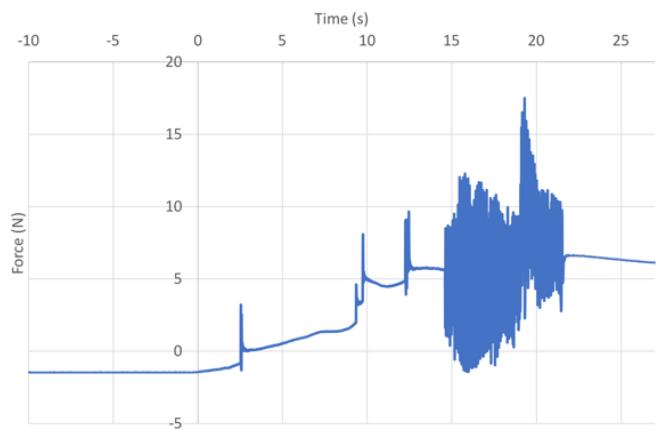

b)

Figure 7: The profiles of joystick signals and forces during cutting test, a) joystick axes signals, $x$ : blue solid, y: orange dotted, z: grey dashed, and b) force signal from the forcesensitive surface. Cutting from time 0 .

As can be seen in Figure 7, the cutting produces side forces around 5-10 $\mathrm{N}$ on the cauliflower. The cutting took about $15-20 \mathrm{~s}$ in this test due to the constrained mobility of the arm. Typically $<10 \mathrm{~s}$ is possible with the current setup. A test was also done to make sure the arm could grasp an actual cauliflower. This test was meant to evaluate whether the arm's geometry had a suitable range to reach a cauliflower and position itself to grasp it (Figure 8). One of the main goals of the lifting test sequence was to ascertain if the picking end-effector can lift the payload of a cauliflower curd and a part of its foliage. See an example lifting sequence in Figure 9. 


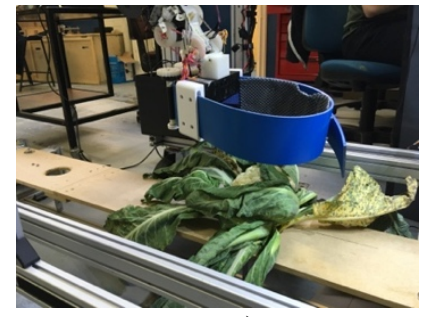

a)

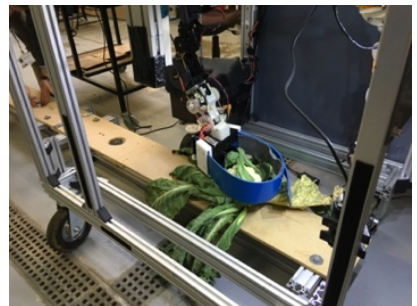

b)

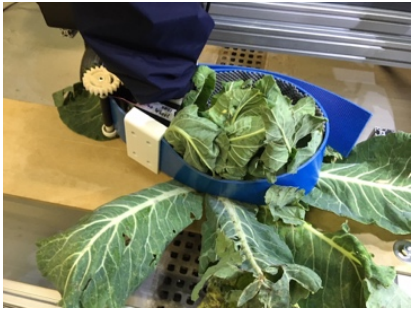

c)

Figure 8: a) initial position, b) approach and c) final grasping position reached.

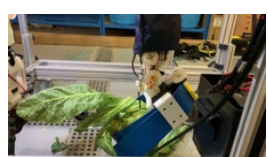

a)

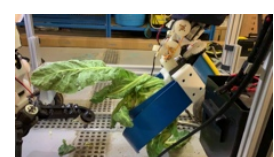

b)

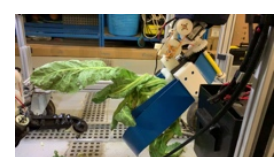

c)

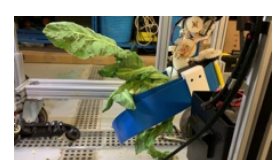

d)

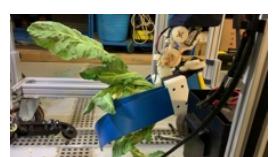

e)

Figure 9: a lifting test sequence (a through e). The test was started with the cauliflower end-effector already closed around the curd, lift and angle it upwards.

\section{Discussion}

While initial results are promising, there is considerable development needed to approach autonomous operation of the platform. So far only approaches to fitting and adjusting lighting conditions and manually tuning classic computer vision algorithms have been implemented. There is good reason to believe that this could be done: If one deploys a platform that is completely covered and with artificially controlled light, identification can likely be performed on a given variety of cauliflowers fairly reliably. In the tests performed so far, the computer vision approach seemed to work well apart from some sensitivity to light (Figure 4). However, this is not expected to be robust between different varieties. A complementary approach is to explore recent advances in deep learning for image processing, such as Convolutional Neural Networks (CNN) (Krizhevsky et al. 2012). Deep learning is also increasingly being applied in agriculture (Kamilaris and Prenafeta-Boldú, 2018), and such techniques have a potential for improving the performance of both maturity classification and curd detection. A sizable dataset of different varieties is needed to be able to apply such techniques. This is part of the future work for this project. Furthermore, the arms are being evolved to be able to better sustain the larger payload of the cauliflowers, including improved cutters and grippers. Different concepts of operation are also being explored with producers, ranging from mounting multiple picking cells on existing tractors, to swarms of self-driving platforms.

\section{Conclusions}

This paper described a proof-of-concept platform for bi-manual robotic cauliflower harvesting. The platform can currently perform the tasks of: 1) identifying the approximate cauliflower maturity for visible curds, 2) localising and grasping the cauliflower curd, and 3) cutting it at a desired location along the stem. For these tasks, it relies on computer vision algorithms for identifying a cauliflower suitable for picking, and passively compliant robotic arms for dealing gracefully with possible collisions with the ground and plant material. These are the first steps towards a fully autonomous and selective cauliflower harvester, which should be run as an integrated system. The 
system architecture is designed to also be adaptable to other similar crops, by replacing the modular end-effectors and the vision/control algorithms.

\section{Acknowledgements}

This work presented here was part of the Automated Brassica harvesting in Cornwall (ABC project), a sub-project of Agritech Cornwall, part-funded by the European Regional Development Fund $(E R D F)$, and with match-funding from Cornwall Council. The work was also partially supported by CNPq Brazil (scholarship 232590/2014-1). Thanks to the Cornish industrial partners, including Riviera Produce (Hayle, UK), Teagle Machinery Ltd (Truro, UK), Robotriks (Par, UK) and CNC Design Ltd (St Columb Major, UK).

\section{References}

Bac, C.W., Hemming, J., van Tuijl, B.A.J., Barth, R., Wais, E. and van Henten, E.J., 2017. Performance evaluation of a harvesting robot for sweet pepper. Journal of Field Robotics, 34(6), pp. 1123-1139.

Davidson, J.R., Silwal, A., Hohimer, C.J., Karkee, M., Mo, C. and Zhang, Q., 2016, October. Proof-of-concept of a robotic apple harvester. In International Conference on Intelligent Robots and Systems (IROS), IEEE/RSJ (pp. 634-639). IEEE.

Intel. 2018. "Overview of the Intel ${ }^{\circledR}$ RealSense ${ }^{\mathrm{TM}}$ Depth Camera." 2018. Accessed December $5^{\text {th }}$ 2018. https://software.intel.com/en-us/realsense/d400.

Kamilaris, A. and Prenafeta-Boldú, F.X., 2018. Deep learning in agriculture: A survey. Computers and Electronics in Agriculture, 147, pp. 70-90.

KMS projects. 2018. "“Breakthrough' automated broccoli harvester turns to FANUC for a helping hand." Accessed December $9^{\text {th }}$ 2018. https://www.fanuc.eu/uk/en/whowe-are/news/uk-kms-automated-harvester-08-2018

Krizhevsky, A., Sutskever, I. and Hinton, G.E., 2012. Imagenet classification with deep convolutional neural networks. In Advances in neural information processing systems. Neural Information Processing Systems Foundation, pp. 1097-1105.

Kusumam, K., Krajník, T., Pearson, S., Duckett, T. and Cielniak, G., 2017. 3D-vision based detection, localization, and sizing of broccoli heads in the field. Journal of Field Robotics, 34(8), pp. 1505-1518.

ROS. n.d. "Flexbe - ROS Wiki." Accessed December 5, 2018. http://wiki.ros.org/flexbe

Stoelen, M.F., Bonsignorio, F., Cangelosi, A., 2016. Co-exploring Actuator Antagonism and Bio-inspired Control in a Printable Robot Arm, In: Tuci, E., Giagkos, A., Wilson, M., Hallam, J. (Eds.), From Animals to Animats 14. Basel, Switzerland: Springer International Publishing, pp. 244-255.

Stoelen, M.F. et al. 2018. "China Robot Harvest++." Accessed December $9^{\text {th }} 2018$. http://www.agritechinchina.org/LG017

Sucan, I.A. and Chitta, S. 2018. "MoveIt!". Accessed December $12^{\text {th }} 2018$. http://moveit.ros.org

University of Plymouth. 2018. "University spinout signs agreement to field test harvesting robots". Accessed December 5, 2018. https://www.plymouth.ac.uk/news/university-spinout-signs-agreement-to-fieldtest-harvesting-robots

Wageningen University. 2018. "Robotic Broccoli Harvester" Accessed December 5, 2018. https://www.wur.nl/en/project/Robotic-Broccoli-Harvester.htm 